INPLASY

PROTOCOL

To cite: Fu et al. Bevacizumab for adult patients of newly diagnosed and recurrent glioblastoma: A Systematic Review and Meta-Analysis. Inplasy protocol 202070074. doi:

10.37766/inplasy2020.7.0074

Received: 16 July 2020

Published: 16 July 2020

Corresponding author: Minjie Fu

16307110106@fudan.edu.cn

Author Affiliation:

Huashan Hospital

Support: No financial support.

Review Stage at time of this submission: Data extraction.

Conflicts of interest:

We declare we have no conflicts of interest in the study.

\section{Bevacizumab for adult patients of newly diagnosed and recurrent glioblastoma: A Systematic Review and Meta-Analysis}

Fu, MJ1; Hua, W2.

Review question / Objective: P: Adult patients of newly diagnosed and recurrent glioblastoma; I: Bevacizumab combined with conventional therapy; C: Conventional therapy; O: OS, PFS; S: RCT.

Condition being studied: Glioblastoma is a kind of malignant solid tumors with poor prognosis. The standard treatment now is surgical resection combined with radiotherapy and temozomide(TMZ). Since glioblastoma is hyperemic activation of VEGFA and HIF activation is upregulated, it might be a good candidate for anti-angiogenesis treatment. And Bevacizumab (BEV) is a humanized monoclonal antibody against the VEGF ligand, which has showed favorable therapeutic effects on some solid types. Our aim was to review the benefit of the Bevacizumab on GBM in the terms of PFS and OS.

INPLASY registration number: This protocol was registered with the International Platform of Registered Systematic Review and Meta-Analysis Protocols (INPLASY) on 16 July 2020 and was last updated on 16 July 2020 (registration number INPLASY202070074).

\section{INTRODUCTION}

Review question / Objective: P: Adult patients of newly diagnosed and recurrent glioblastoma; I: Bevacizumab combined with conventional therapy; C: Conventional therapy; O: OS, PFS; S: RCT.

Condition being studied: Glioblastoma is a kind of malignant solid tumors with poor prognosis. The standard treatment now is surgical resection combined with radiotherapy and temozomide(TMZ). Since glioblastoma is hyperemic activation of VEGFA and HIF activation is upregulated, it might be a good candidate for antiangiogenesis treatment. And Bevacizumab (BEV) is a humanized monoclonal antibody against the VEGF ligand, which has showed favorable therapeutic effects on some solid 
types. Our aim was to review the benefit of the Bevacizumab on GBM in the terms of PFS and OS.

\section{METHODS}

Search strategy: We performed a comprehensive literature search via PubMed(updated through June 2020) using the search words "bevacizumab", "avastin" and "glioblastoma". No language or date limitations were imposed. An independent search using the citation database "Web of Science" was performed to ensure that all relevant papers were included in the metaanalysis. Efforts were also made to contact the investigators and the manufacturer of bevacizumab when relevant data were not clear.

Participant or population: Adult patients of newly diagnosed and recurrent glioblastoma.

Intervention: Bevacizumab with conventional therapy.

Comparator: Conventional therapy without bevacizumab.

Study designs to be included: Randomized controlled trials.

Eligibility criteria: (1) Randomized trials recruiting adult patients with newly diagnosed or recurrent glioblastoma; (2) Studies analyzing patients treated with bevacizumab (alone or combined with conventional therapy) versus conventional therapy alone; (3) HR for OS and PFS had to be reported or could be computed from the data presented.

Information sources: Pubmed database, contact with authors.

Main outcome(s): For some duplicated studies, the last available update of each trial was considered as the original source. We extracted details regarding the number of patients, mean age, treatment information and hazard ratio (HR) for progression-free survival and overall survival. For those studies in the absence of hazard ratio, we calculated the HR indirectly with the Kaplan-Meier curve by excel tools(Tierney et al., 2007). The treatment information included bevacizumab alone or in combination with other medicine, dose and treatment cycle.

Quality assessment / Risk of bias analysis: Publication bias was assessed by visual inspection of funnel plots for study size against treatment effect. We also carried out a sensitivity analysis by iteratively recalculating the pooled HR estimate after exclusion of each single study to evaluate whether the pooled estimates should be included in the meta-analysis. Subgroup analyses were performed according to the stage of glioblastoma (newly diagnosed or recurrent).

Strategy of data synthesis: The pooled analysis calculations and the generation of forest plots were accomplished using the Review Manager 5.4 (https: / / training.cochrane.org/). Pooled estimates of HRs were obtained using a random or fixed effect model according to the heterogeneity between trials. The derived results were reported as a conventional meta-analysis forest plot.

Subgroup analysis: Subgroup analyses were performed according to the stage of glioblastoma (newly diagnosed or recurrent).

Sensibility analysis: We carried out a sensitivity analysis by iteratively recalculating the pooled HR estimate after exclusion of each single study to evaluate whether the pooled estimates should be included in the meta-analysis.

Language: No limitations.

Country(ies) involved: China.

Keywords: bevacizumab; glioblastoma; Clinical trial; meta-analysis.

Contributions of each author:

Author 1 - Minjie Fu.

Author 2 - Wei Hua. 